\title{
Excision of abdominal wall recurrence of uterine leiomyosarcoma: A case report
}

\author{
Sahana Punneshetty ${ }^{1}$, Rachel G Chandy ${ }^{2}$, Geley Ete ${ }^{3}$, Suchita Chase ${ }^{4}$, Anjana Joel', Abraham Peedicayil ${ }^{2}$ \\ From ${ }^{1}$ Senior Resident, ${ }^{2}$ Professor, Department of Gynaecologic Oncology, ${ }^{3}$ Associate Professor, Department of Plastic Surgery, ${ }^{4}$ Professor, Department \\ of General Surgery, ${ }^{5}$ Assistant Professor, Department of Medical Oncology, CMC, Vellore, Tamil Nadu, India
}

\begin{abstract}
The literature on management of recurrent/metastatic uterine leiomyosarcomas is limited and metastasis to the abdominal wall is very rare. We operated on a 46-year-old woman who had a total abdominal hysterectomy elsewhere for a uterine tumor and presented with recurrence to the anterior abdominal wall, 9 months after the primary surgery. There were two large ulcerated abdominal wall masses. The challenge after complete excision of the tumor was to reconstruct the abdominal wall. We were able to close the defect with a dual mesh and a fasciocutaneous flap from the anterolateral thigh. She received adjuvant chemotherapy with gemcitabine and docetaxel. At the completion of chemotherapy, she was well and apparently disease free. She recurred in the left inguinal node after 9 months but responded to oral pazopanib followed by chemotherapy. This case report highlights the rare possibility of abdominal wall metastasis after initial surgery for leiomyosarcoma. It shows that good quality of life can be achieved by a multidisciplinary treatment of abdominal wall metastasis of uterine leiomyosarcoma.
\end{abstract}

Key words: Abdominal metastasis, Case report, Reconstruction, Uterine leiomyosarcoma

$\mathrm{U}$ terine leiomyosarcomas (LMS) constitute $1 \%$ of all uterine malignancies but account for $60 \%$ of uterine sarcomas. They usually spread by hematogenous route, unlike endometrial carcinomas. The most common metastatic sites are the lung, peritoneum, bones, and liver. Other sites include pelvic lymph nodes, soft tissue, thyroid, salivary gland, heart, pancreas, adrenal gland, bowel, and breast. The recurrence rate is $45-75 \%$ and the site is often distant [1]. Abdominal wall recurrence is rare [2]. Patients with uterine leiomyosarcoma have a $25 \%$ (Stage II) to $75 \%$ (Stage IA) 5-year survival rate [3]. The management of the recurrent or metastatic disease is a challenge as most of them are refractory to chemotherapy or radiation therapy.

\section{CASE REPORT}

A 46-year-old unmarried woman presented to us with a nonhealing ulcerative abdominal wall mass of 3 months duration. She was hypertensive on telmisartan $40 \mathrm{mg}$ daily. Her father had cancer of the lung.

She had had a total abdominal hysterectomy done elsewhere, a year earlier, for heavy menstrual bleeding. Histopathology was atypical leiomyoma with myxoid degeneration. No further

\section{Access this article online}

Received - 15 March 2021

Initial Review - 31 March 2021

Accepted - 08 April 2021

DOI: 10.32677/IJCR.2021.v07.i04.009 therapy was given. After surgery, the patient was well for about 8 months and then noticed an abdominal tingling sensation and non-healing ulcers at the surgical scar for which she underwent incision and drainage multiple times. She then noticed a swelling at the previous scar. The timeline is shown in Table 1. In view of a non-healing ulcer, she underwent a biopsy of the lesion which was reported as a poorly differentiated malignant tumor positive for vimentin, SMA, and $\mathrm{Ki} 67$, and was referred to a tertiary center for further management.

On admission, general examination showed that there was no pallor or lymphadenopathy. Her pulse rate was $104 / \mathrm{min}$, blood pressure was 110/60 $\mathrm{mmHg}$, and functional status was Eastern Cooperative Oncology Group 1. She had two large $(9 \mathrm{~cm} \times 7 \mathrm{~cm}$ and $7 \mathrm{~cm} \times 5 \mathrm{~cm}$ ) adjacent abdominal wall lumps (Fig. 1a) with surface ulcers. There were two large lobulated heterogeneously enhancing soft-tissue lesions with large areas of central necrosis in the lower anterior abdominal wall, centered in the subcutaneous and muscular plane with posterior extension into the pre-peritoneal space. Vulva and vagina were normal. Rectal examination was also normal.

Her biopsy slides were reviewed by our pathologist. Her positron emission tomography and computed tomography scan (Fig. 1b) showed that lesions were confined to the abdominal wall. A primary abdominal wall tumor was considered less likely than a recurrence from uterine sarcoma. The slides were reviewed at our hospital and were reported as high-grade sarcoma. The clinical details and

Correspondence to: Abraham Peedicayil, Department of Gynaecologic Oncology, CMC Hospital, Vellore - 632 004, Tamil Nadu, India. E-mail: apeedicayil@yahoo.com

(C) 2021 Creative Commons Attribution-NonCommercial 4.0 International License (CC BY-NC-ND 4.0). 
Table 1: Timeline of a case of leiomyosarcoma metastasis to abdominal wall

January 2019 Heavy menses - underwent total abdominal hysterectomy elsewhere. Histopathology showed atypical leiomyoma - no adjuvant treatment

September 2019 Abdominal ulceration - "drainage of abscess"

December 2019 Abdominal wall swelling growing rapidly

January 2020

Presented to us and underwent excision of mass with pelvic peritoneal nodules and repair of defect with dual mesh and anterolateral fasciocutaneous flap

April 2020 Wounds healed well and patient well on review

June 2020 Completed 6 cycles of gemcitabine+docetaxel

September 2020

Left inguinal nodal swelling/recurrence treated with tab. pazopanib $800 \mathrm{mg}$ daily

October 2020 Inguinal swelling subsided; patient well January 2021 Enlargement and ulceration of inguinal swelling. Started on injection eribulin.
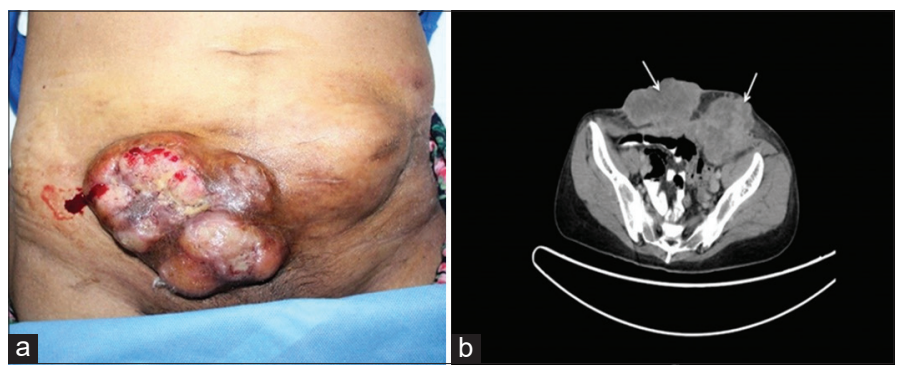

Figure 1: Abdominal masses. (a) On inspection. (b) On computerized tomography

images were discussed in our multidisciplinary tumor board and she was planned for upfront surgery followed by chemotherapy.

She underwent excision of abdominal wall nodules (Fig. 2a), bilateral oophorectomy, and removal of rectosigmoid nodules. Reconstruction of lower anterior abdominal wall with mesh (Fig. 2b) and pedicled left anterolateral thigh fasciocutaneous flap was done on January 24, 2020 (Figs. 3a and b, 4a). The left thigh flap donor site was resurfaced with a split skin graft. She was gradually mobilized and was discharged on post-operative day 14. Histopathology showed necrosis, mitotic activity $>10 / 10 \mathrm{HPF}$, and resected margins were free of tumor. Bilateral ovaries and fallopian tubes were also free of tumor. The final report was a poorly differentiated sarcoma of the abdominal wall.

She received 5 cycles of adjuvant chemotherapy with injection gemcitabine $1000 \mathrm{mg} / \mathrm{m}^{2}$ on day 1 and injection gemcitabine $1000 \mathrm{mg} / \mathrm{m}^{2}$ and injection docetaxel $75 \mathrm{mg} / \mathrm{m}^{2}$ on day 8 . The patient was reviewed and found to be well 4 months after surgery (Fig. 4b). Nine months after surgery, she had a recurrence in the left groin, and she was started on oral pazopanib in September 2020. As the response was inadequate, she was then started on intravenous eribulin in January 2021.

\section{DISCUSSION}

LMSs are the most common sarcomas accounting for $1-2 \%$ of all uterine malignancies with an incidence of $0.3-0.4 / 100,000$

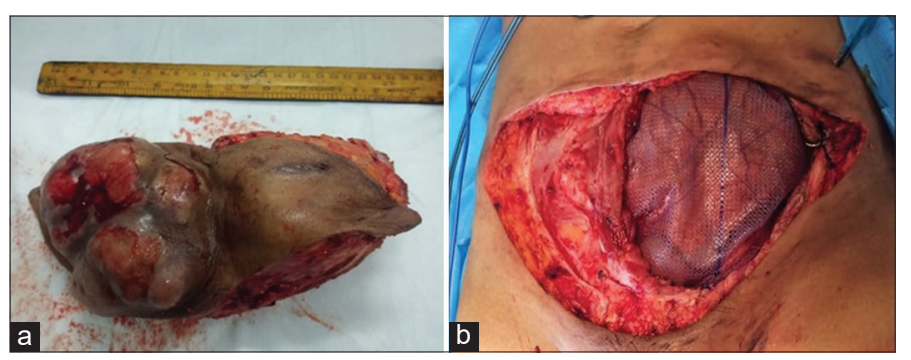

Figure 2: Surgical findings (a) Excised abdominal masses. (b) Mesh placement to repair defect in rectus sheath

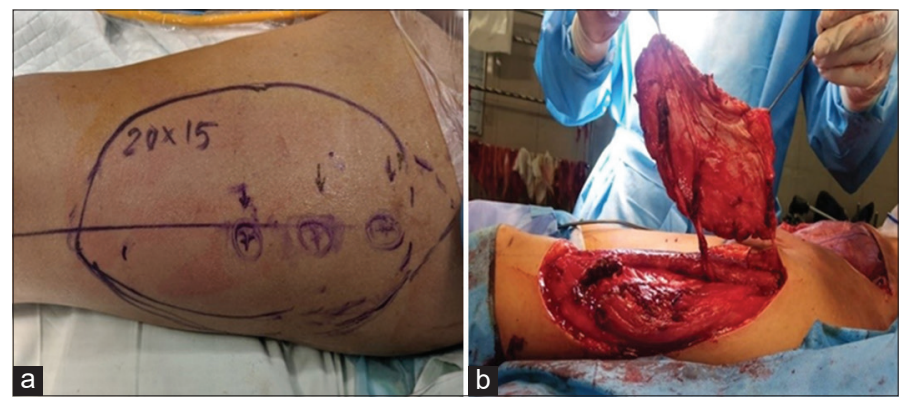

Figure 3: Anterolateral thigh fasciocutaneous flap (a) Flap markings on the left thigh with three identified perforators. (b) Flap harvested on descending branch of lateral circumflex femoral artery



Figure 4: Anterior abdominal wall reconstruction. (a) To cover the mesh after inserting of flap. (b) Well-settled flap, 4 months after surgery

women per year [2]. The most common age of presentation is $45-55$ years with a median age of 52 years. The common symptoms include abnormal vaginal bleeding (56\%), palpable pelvic mass $(54 \%)$, and pelvic pain $(22 \%)$. They are more likely to have early hematogenous spread. Most of the tumors are aggressive with a poor prognosis and high local recurrence rate.

They usually arise de novo from uterine smooth muscle. Pelvic radiation [4] and the use of combined hormone replacement therapy in postmenopausal women for 5 years or longer are also associated with an increased risk [5]. Uterine curetting is positive in only $10-20 \%$ [6]. The tumors are usually larger than $10 \mathrm{~cm}$ in size. The cut surface is typically soft, bulging, fleshy, necrotic, and hemorrhagic with irregular margins.

Uterine LMSs consist of highly cellular, spindle-shaped smooth muscle cells, mitotic figures, and coagulative necrosis. The histological subtypes include spindled, epithelioid, and myxoid types. The spindled variant accounts for most cases with a very high mitotic index [7]. Tumors are graded into low grade and high grade depending on cellular atypia, mitotic index, and the presence of necrosis which are the main prognostic factors for 
recurrence. The most common immunohistochemistry markers are desmin, h-caldesmon, smooth muscle actin, and histone deacetylase. They also express estrogen receptors, progesterone receptors, and androgen receptors. LMSs express higher levels of Ki-67 than leiomyomas. The genetic profile suggests genomic instability which is a hallmark of uterine sarcomas. The presence of smooth muscle actin is nearly uniform and desmin positivity is usual. This along with the lack of KIT expression separates leiomyosarcoma from the gastrointestinal stromal tumors, an important problem in abdominal soft tissues [8].

Surgery is the mainstay of treatment and includes total abdominal hysterectomy with bilateral salpingo-oophorectomy and debulking of any tumor outside the uterus including enlarged nodes. Stage is the most powerful prognostic factor. Spindle cell LMSs recur within 2 years compared to other variants which recur late. There is sparse literature on the management of recurrent uterine leiomyosarcoma. Studies have demonstrated that the patients with recurrent uterine sarcomas who underwent secondary cytoreductive surgery had longer overall survival and disease-free intervals following the initial relapse when compared with those treated with chemotherapy or radiotherapy alone. Surgery for extrapulmonary recurrences of uterine sarcomas is limited [9].

Chemotherapy with or without palliative radiation therapy is generally recommended for patients with Stages II and III disease, incompletely resected, or metastatic disease [10]. The chemotherapeutic agents used for recurrent disease are fixed-dose gemcitabine and docetaxel, single-agent doxorubicin, paclitaxel, trabectedin, eribulin, megestrol acetate, aromatase inhibitors, and pazopanib. Adjuvant radiation therapy is considered for patients with high recurrence risk as it has shown to reduce the pelvic relapse rate by $50 \%$. Cutaneous metastasis of uterine leiomyosarcoma has been reported earlier [11]. Secondary cytoreduction may be considered, as neither chemotherapy nor radiation improved outcomes in recurrent disease [12]. Secondary cytoreduction is associated with prolonged survival in patients with isolated disease recurrence and long progression-free interval from the primary disease $[9,13]$.

\section{CONCLUSION}

The management of recurrent LMSs and advanced-stage disease depends on resectability. Surgery and metastasectomy, in properly selected patients, may improve quality of life and survival. Multimodality treatment and palliation should also be considered.

\section{ACKNOWLEDGMENT}

Ms. B for allowing us to publish her story.

\section{REFERENCES}

1. Reed N. The management of uterine sarcomas. In: Ayhan A, Reed N, Gultekin M, Dursun P, editors. Text Book of Gynaecology. $3^{\text {rd }}$ ed. Turkey: Guines Publishing; 2016. p. 543-9.

2. Harlow BL, Weiss NS, Lofton S. The epidemiology of sarcomas of the uterus. J Natl Cancer Inst 1986;76:399-402.

3. Abeler VM, Røyne O, Thoresen S, Danielsen HE, Nesland JM, Kristensen GB. Uterine sarcomas in Norway: A histopathological and prognostic survey of a total population from 1970 to 2000 including 419 patients. Histopathology 2009;54:355-64.

4. Skubitz KM, Adamo DR. Sarcoma. Mayo Clin Proc 2007;82:1409-32.

5. Felix AS, Cook LS, Gaudet MM, Rohan TE, Schouten LJ, Setiawan VW, et al. The etiology of uterine sarcomas: A pooled analysis of the epidemiology of endometrial cancer consortium. Br J Cancer 2013;108:727-34.

6. Dinh TV, Woodruff JD. Leiomyosarcoma of the uterus. Am J Obstet Gynecol 1982;144:817-23.

7. Pelmus M, Penault-Llorca F, Guillou L, Collin F, Bertrand G, Trassard M, et al. Prognostic factors in early-stage leiomyosarcoma of the uterus. Int J Gynecol Cancer 2009; 19:385-90.

8. Miettinen M. Smooth muscle tumors of soft tissue and non-uterine viscera: Biology and prognosis. Mod Pathol 2014;27 Suppl 1:S17-29.

9. Nakamura K, Kajiyama H, Utsumi F, Suzuki S, Niimi K, Sekiya R, et al. Secondary cytoreductive surgery potentially improves the oncological outcomes of patients with recurrent uterine sarcomas. Mol Clin Oncol 2018;8:499-503.

10. Andrea R. Role of chemotherapy in cancers of the uterus. In: Annunziata C, Chu CS, Rubin SC, editors. Chemotherapy for Gynecologic Cancers: Society of Gynecologic Oncology Handbook. $3^{\text {rd }}$ ed. Lake Zurich: Schneider Graphics; 2017. p. 160-82.

11. Barbetakis N, Paliouras D, Asteriou C, Samanidis G, Kleontas A, Anestakis D, et al. Cutaneous skull metastasis from uterine leiomyosarcoma: A case report. World J Surg Oncol 2009;7:45.

12. Giuntoli RL 2 ${ }^{\text {nd }}$, Metzinger DS, DiMarco CS, Cha SS, Sloan JA, Keeney GL, et al. Retrospective review of 208 patients with leiomyosarcoma of the uterus: Prognostic indicators, surgical management, and adjuvant therapy. Gynecol Oncol 2003;89:460-9.

13. Giuntoli RL 2 ${ }^{\text {nd }}$, Garrett-Mayer E, Bristow RE, Gostout BS. Secondary cytoreduction in the management of recurrent uterine leiomyosarcoma. Gynecol Oncol 2007;106:82-8.

Funding: None; Conflicts of Interest: None Stated.

How to cite this article: Punneshetty S, Chandy RG, Ete G, Chase S, Joel A, Peedicayil A. Excision of abdominal wall recurrence of uterine leiomyosarcoma: A case report. Indian J Case Reports. 2021;7(4):152-154. 